\title{
Linear and circular polarization of comet C/2009 P1 (Garradd)
}

\author{
N. N. Kiselev ${ }^{1}$, V. K. Rosenbush ${ }^{1}$, V. L. Afanasiev², S. V. Kolesnikov ${ }^{3}$, S. V. Zaitsev ${ }^{1}$, and D. N. Shakhovskoy ${ }^{4}$ \\ ${ }^{1}$ MAO NAS Ukraine, 27 Zabolotnoho str., 03680 Kyiv, Ukraine \\ ${ }^{2}$ Special Astrophysical Observatory, RAS, Nizhnii Arkhyz, Karachai-Cherkessian Republic, 357147 Russia \\ ${ }^{3}$ Observatory of the Odessa National University, Shevchenko Park, 65014 Odessa, Ukraine \\ ${ }^{4}$ Crimean Astrophysical Observatory, Nauchny, 98409, Crimea, Ukraine
}

(Received October 31, 2012; Revised May 23, 2013; Accepted May 24, 2013; Online published October 24, 2013)

\begin{abstract}
We report measurements of linear and circular polarization for the recent comet C/2009 P1 (Garradd) during its approach to the Earth in 2011-2012. Aperture photoelectric observations were carried out on July 29, 2011-April 22, 2012 in the R and WR wide-band filters at the 2.6-m telescope of the Crimean Astrophysical Observatory (Ukraine). Spectropolarimetric measurements of linear polarization in the range 3800-8000 $\AA$ and imaging circular polarimetry in the comet continuum filter $\left(\lambda_{0}=6840 / 90 \AA\right)$ were also carried out with the SCORPIO-2 focal reducer at the 6-m telescope of the Special Astrophysical Observatory (Russia) on February 2-April 21, 2012. The degree of polarization of light scattered by comet Garradd changes from $-2.2 \pm 0.2 \%$ to $4.9 \pm 0.2 \%$ at phase angles $13.7^{\circ}$ and $35.9^{\circ}$, respectively. These values are in a good agreement with those for dusty comets at the respective phase angles. Left-handed (negative) circular polarization of comet Garradd in the continuum filter was identified. The values of Pc vary noticeably over the coma in the range from -0.03 to $-0.08 \%$ and from -0.05 to $-0.3 \%$ on February 14 and April 21, respectively. These results confirm our previous conclusion that the observed circular polarization for comets is predominantly left-handed.
\end{abstract}

Key words: Comets, comet C/2009 P1 (Garradd), linear and circular polarization.

\section{Introduction}

Polarimetry is a powerful tool for probing the physical properties of cometary dust particles; see numerous examples in Mishchenko et al. (2010) and references therein. One of the ways of studying the properties of cometary dust is a comparison of the polarization phase angle dependence for different comets. For this purpose, we created the Database of Comet Polarimetry (Kiselev et al., 2010) which currently contains polarimetric data for 64 comets. In recent years, new data have been collected for a number of comets. Here, we present the results of linear and circular polarimetric observations of the new comet $\mathrm{C} / 2009$ P1 (Garradd) (hereafter Garradd) during its approach to the Earth in 2011-2012.

Aperture polarimetric observations of comets in broadband filters are still more frequent because they allow one to observe fainter objects. Such observations using narrowband cometary filters for bright comets are very effective (see, e.g. Kiselev and Velichko, 1997, 1998), although they lose in the spatial resolution as compared with imaging polarimetry. For distant comets, polarimeters with CCD detectors have an advantage only in their penetrating power over photoelectric polarimeters, because observations of fainter comets are carried out with broad-band filters, and the data refer to the average values for the virtual apertures. In addition to aperture polarimetry of comet Garradd, we

Copyright (C) The Society of Geomagnetism and Earth, Planetary and Space Sciences (SGEPSS); The Seismological Society of Japan; The Volcanological Society of Japan; The Geodetic Society of Japan; The Japanese Society for Planetary Sciences; TERRAPUB.

doi:10.5047/eps.2013.05.014 present the results of spectropolarimetric observations of the comet with a long slit. Such observations remain scarce (see, e.g. Harrington et al., 2006). The spatial resolution of spectropolarimetric observations of comets can only be high in the region of coma, determined by the size of the slit, but the high spectral resolution reveals the great advantage of such observations, which is especially important taking into account the complexity of cometary spectra.

Unfortunately, to date, observations of comets are usually performed with polarimeters designed to study star-like objects, and there are practically no polarimeters optimally designed for the study of comets. It is obvious that, under such circumstances, combining instruments with different designs can yield improved results. This is exactly what we have done in our work.

\section{Observations}

Measurements of linear polarization for comet Garradd were carried out at the 2.6-m Shain telescope of the Crimean Astrophysical Observatory (Ukraine) with a single-channel photopolarimeter (Shakhovskoy and Efimov, 1972; Mishchenko et al., 2010) during seven nights from July 29, 2011, through April 22, 2012. The R wideband filter $\left(\lambda_{0}=6400 / 800 \AA\right)$ and the WR filter (5500$7500 \AA)$ in combination with a 15 arcsec diaphragm were used. The photopolarimeter consists of a motionless Glan prism installed immediately behind an achromatic quarterwave phase plate rotating with a frequency of $\sim 33 \mathrm{~Hz}$. This enables all four Stokes parameters to be measured quasi-simultaneously according to a modulation formula (Serkowski, 1974). The principle of synchronous detec- 
tion is used. The intensity components used to compute the Stokes parameters $Q, U, V$ are selected by eight virtual counters (one physical counter) switched on sequentially and accumulate the signal for 16 position angles of each rotation of the phase plate $\left(0^{\circ}-22.5^{\circ}, 22.5^{\circ}-45, \ldots, 337.5^{\circ}-\right.$ $360^{\circ}$ ). The time of a single integration was $\sim 4 \mathrm{~s}(133$ rotations of the quarter-wave plate). One exposure of the comet consisted of 16 integrations. The cumulative observation consisted of 15 to 60 exposures, depending on the brightness of the comet and the observation conditions. The sky background was measured several times during the observations of the comet. Aperture measurements with a large diaphragm are not effective for measuring the circular polarization for comets, so we did not use these observations to determine the Stokes parameter $V$. The normalized Stokes parameters of linear polarization for a comet are given by the following expressions:

$$
\begin{aligned}
& q=314.16\left(N_{1}+N_{2}-N_{3}-N_{4}+N_{5}+N_{6}-N_{7}-N_{8}\right) / N, \\
& u=314.16\left(-N_{1}+N_{2}+N_{3}-N_{4}-N_{5}+N_{6}+N_{7}-N_{8}\right) / N,
\end{aligned}
$$

where $N_{\mathrm{i}}$ is the number of photoelectron pulses accumulated by the $i$ th counter during the full time of the observation of the comet (without sky background), and $N$ is the number of photoelectron pulses accumulated by the eight counters. The instrumental polarization parameters qinst and uinst were determined from observations of unpolarized standard stars. Correction for the zero-point of the position angle of the polarization plane $\Delta \theta$ was obtained using observations of standard stars with well-known large interstellar polarization. Standard stars were taken from Serkowski (1974) and Hsu and Breger (1982). The instrumental parameters qinst and uinst did not exceed $0.15 \pm$ $0.05 \%$ in the filters used and were excluded from the polarization parameters for the comet. The zero-point of the position angle of the polarization plane was stable within $0.2^{\circ}$. The final average values of the degree of linear polarization and position angle of the polarization plane of comet are calculated according to the following expressions:

$$
\begin{aligned}
P & =\sqrt{u^{2}+q^{2}}, \\
\theta & =\frac{1}{2} \arctan \frac{u}{q}+\Delta \theta .
\end{aligned}
$$

The random polarization errors were estimated from the following formula (Shakhovskoy and Efimov, 1972):

$$
\sigma_{0 P}=\sigma_{0 u}=\sigma_{0 q}=\frac{\pi 100}{2} \sqrt{\frac{1}{N}\left(1+\frac{1+\tau}{R}\right)},
$$

where $\tau$ is the ratio of the accumulation times for the comet and the background sky, and $R$ is the ratio of the average pulse rates for the comet and the background sky. Another estimate of the errors is obtained as the standard deviation of random variables for separate measurements of the Stokes parameters $q_{\mathrm{i}}$ and $u_{\mathrm{i}}$ (Shakhovskoy and Efimov, 1972). The greater of the two average errors (from the statistics of photoelectrons and the dispersion of polarization parameters) serves as the estimate of the error of polarization parameters $\sigma_{q \text {,com }}$ and $\sigma_{u \text {,com }}$. The final errors of comet polarization parameters include the errors of instrumental polarization $\sigma_{q \text {,inst }}$ and $\sigma_{u \text {,inst }}$ :

$$
\begin{aligned}
\sigma_{q} & =\sqrt{\sigma_{q, \mathrm{com}}^{2}+\sigma_{q, \mathrm{inst}}^{2}}, \\
\sigma_{u} & =\sqrt{\sigma_{u, \mathrm{com}}^{2}+\sigma_{u, \mathrm{inst}}^{2}}, \\
\sigma_{P} & =\sqrt{0.5\left(\sigma_{u}^{2}+\sigma_{q}^{2}\right)}, \\
\sigma_{\theta} & =28.65 \frac{\sigma_{P}}{P} .
\end{aligned}
$$

The results of the measurements of linear polarization for comet Garradd are given in Table 1, which lists: the date of observations, the degree of linear polarization $P_{\mathrm{r}}$ with respect to the scattering plane and its mean error $\sigma_{P}$, the position angle of the polarization plane $\theta$ and its mean error $\sigma_{\theta}$, the size of the area (diaphragm) projected at the comet, the position angle of the scattering plane $\phi$, the phase angle $\alpha$, the heliocentric distance $r$ and the geocentric distance $\Delta$ of the comet, and the spectral filter. The values $P_{\mathrm{r}}$ are linked to the quantities $P$ through the relation $P_{\mathrm{r}}=P \cos 2\left(\theta-\left(\phi \pm 90^{\circ}\right)\right)$. The sign in the parentheses is chosen to ensure that $0^{\circ} \leq \phi \pm 90^{\circ}<180^{\circ}$ (Chernova et al., 1993).

The long-slit spectropolarimetric measurements of linear polarization in the range $3800-8000$ (the dispersion is $2 \AA$ $\mathrm{px}^{-1}$ in the $\mathrm{V}$ band), as well as imaging circular polarimetry in the RC narrow-band cometary continuum filter $\left(\lambda_{0}=\right.$ $6840 / 90 \AA$ ), were carried out with the SCORPIO-2 focal reducer at the 6-m BTA telescope of the Special Astrophysical Observatory on February 2-April 21, 2012. These were the first measurements of cometary polarization ever attempted with this telescope and this instrument, so we describe these measurements in more detail.

In the spectropolarimetric mode of the instrument, an achromatic half-wave phase plate and a Wollaston prism are installed before a grism. The Wollaston analyzer splits images of the ordinary and extraordinary rays by $5^{\circ}$. For fixed positions of the $\lambda / 2$ phase plate $0^{\circ}, 45^{\circ}, 22.5^{\circ}$, and $67.5^{\circ}$, a series of pairs of spectra $I(\lambda)_{\mathrm{o}}$ and $I(\lambda)_{\mathrm{e}}$ in mutually perpendicular polarization planes is obtained at the exit of the spectrograph. In general, when the orientation of the principal axes of the analyzer $\varphi$ and the vector direction maximum speed phase plate $\theta$ are arbitrary, measured up to the transformation of rotation values of the Stokes parameters $U, Q$ the linear polarization is given by the relations:

$$
\begin{aligned}
& U^{\prime}=0.5\left\{\frac{I(\lambda)_{\mathrm{o}}-I(\lambda)_{\mathrm{e}}}{I(\lambda)_{\mathrm{o}}+I(\lambda)_{\mathrm{e}}}\right\}_{\theta=0^{\circ}}-0.5\left\{\frac{I(\lambda)_{\mathrm{o}}-I(\lambda)_{\mathrm{e}}}{I(\lambda)_{\mathrm{o}}+I(\lambda)_{\mathrm{e}}}\right\}_{\theta=45^{\circ}}, \\
& Q^{\prime}=0.5\left\{\frac{I(\lambda)_{\mathrm{o}}-I(\lambda)_{\mathrm{e}}}{I(\lambda)_{\mathrm{o}}+I(\lambda)_{\mathrm{e}}}\right\}_{\theta=22.5^{\circ}}-0.5\left\{\frac{I(\lambda)_{\mathrm{o}}-I(\lambda)_{\mathrm{e}}}{I(\lambda)_{\mathrm{o}}+I(\lambda)_{\mathrm{e}}}\right\}_{\theta=67.5^{\circ}} .
\end{aligned}
$$

The true values of the Stokes parameters are as follows:

$$
\begin{aligned}
& U=U^{\prime} \cos 2 \varphi-Q^{\prime} \sin 2 \varphi, \\
& Q=U^{\prime} \sin 2 \varphi+Q^{\prime} \cos 2 \varphi .
\end{aligned}
$$

The final degree of linear polarization and the position angle of the plane of polarization were calculated from relations similar to Eq. (2). The long-slit observations were 
Table 1. The results of aperture observations of linear polarization for comet C/2009 P1 (Garradd).

\begin{tabular}{|c|c|c|c|c|c|c|c|c|}
\hline $\begin{array}{c}\text { Date, UT } \\
20011 / 2012\end{array}$ & $\begin{array}{c}P_{\mathrm{r}} \pm \sigma_{P} \\
\%\end{array}$ & $\begin{array}{c}\theta \pm \sigma_{\theta} \\
\operatorname{deg}\end{array}$ & $\begin{array}{c}\text { Area } \\
\mathrm{km}\end{array}$ & $\begin{array}{c}\phi \\
\operatorname{deg}\end{array}$ & $\begin{array}{c}\alpha \\
\operatorname{deg}\end{array}$ & $\begin{array}{c}\mathrm{r} \\
\mathrm{AU}\end{array}$ & $\begin{array}{c}\Delta \\
\mathrm{AU}\end{array}$ & Filter \\
\hline July 29.048 & $-2.21 \pm 0.23$ & $37.6 \pm 2.9$ & 17232 & 211.6 & 13.70 & 2.482 & 1.584 & WR \\
\hline Aug 29.975 & $-0.27 \pm 0.10$ & $110.8 \pm 10.7$ & 15274 & 120.5 & 20.91 & 2.188 & 1.404 & WR \\
\hline Sep 26.786 & $2.37 \pm 0.08$ & $0.7 \pm 1.0$ & 17929 & 89.3 & 30.87 & 1.954 & 1.648 & $\mathrm{R}$ \\
\hline Sep 28.825 & $2.65 \pm 0.25$ & $176.4 \pm 2.7$ & 18179 & 87.6 & 31.14 & 1.938 & 1.671 & $\mathrm{R}$ \\
\hline Oct 26.729 & $2.51 \pm 0.28$ & $153.4 \pm 3.2$ & 21432 & 64.1 & 30.28 & 1.741 & 1.970 & $\mathrm{R}$ \\
\hline Mar 24.924 & $1.67 \pm 0.13$ & $58.6 \pm 2.3$ & 15296 & 147.1 & 28.02 & 1.988 & 1.406 & WR \\
\hline Apr 22.813 & $1.60 \pm 0.17$ & $13.0 \pm 3.1$ & 21606 & 107.7 & 26.71 & 2.237 & 1.986 & WR \\
\hline
\end{tabular}

Table 2. Observational $\log$ of comet C/2009 P1 (Garradd). The 6-m telescope at the SAO.

\begin{tabular}{cccccc}
\hline $\begin{array}{c}\text { Date, } \mathrm{UT} \\
2012\end{array}$ & $\mathrm{r}$ & $\Delta$ & $\alpha$ & Filter/region & Exp \\
\hline Feb 2.085 & $\mathrm{AU}$ & $\mathrm{AU}$ & $\mathrm{deg}$ & $\AA$ & $\mathrm{s}$ \\
Feb 14.089 & 1.647 & 1.533 & 35.87 & $3800-8000$ & 600 \\
Apr 21.833 & 2.228 & 1.963 & 26.81 & $6840 / 90$ & 840 \\
\hline
\end{tabular}

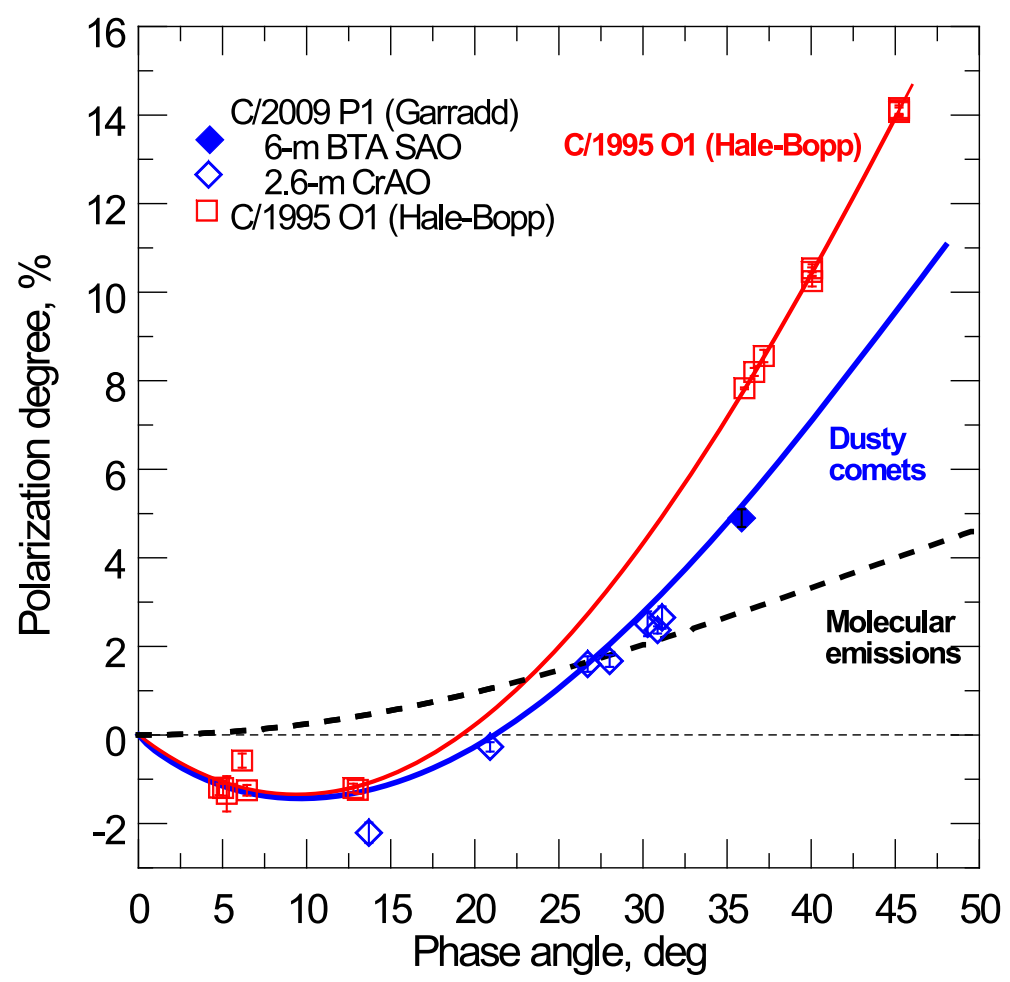

Fig. 1. Phase-angle dependence of linear polarization for comet Garradd compared with the typical polarization for dusty comets, the unusual dusty comet C/1995 O1 (Hale-Bopp), and molecular emissions.

carried out with a $3 \operatorname{arcsec} \times 6$ arcmin slit oriented at a position angle of $217.1^{\circ}$ and thus perpendicular to the extended Sun-comet radius vector on February 2, 2012.

For the measurement of circular polarization, a $\lambda / 4$ phase plate is introduced in the beam for two angles $0^{\circ}$ and $90^{\circ}$. In this case, the circular polarization of the Stokes parameter $V$ is found from the following relation:

$$
V=0.5\left\{\frac{I(\lambda)_{\mathrm{o}}-I(\lambda)_{\mathrm{e}}}{I(\lambda)_{\mathrm{o}}+I(\lambda)_{\mathrm{e}}}\right\}_{\theta=0^{\circ}}-0.5\left\{\frac{I(\lambda)_{\mathrm{o}}-I(\lambda)_{\mathrm{e}}}{I(\lambda)_{\mathrm{o}}+I(\lambda)_{\mathrm{e}}}\right\}_{\theta=90^{\circ}} .
$$

In the direct image mode of the instrument, instead of the spectra $I(\lambda)_{\mathrm{o}}$ and $I(\lambda)_{\mathrm{e}}$, images of the comet obtained in or- dinary and extraordinary rays are used. A detailed description of the instrument and the reduction procedure are given by Afanasiev and Moiseev $(2005,2011)$ and by Afanasiev and Amirkhanyan (2012), respectively. Information about the geometric condition of observations and the exposure times is given in Table 2. We can briefly summarize that the comet was observed in the range of phase angles $13.7^{\circ}-$ $35.9^{\circ}$. The heliocentric and geocentric distances changed within the ranges 1.647-2.492 A.U. and 1.386-1.966 A.U., respectively. 

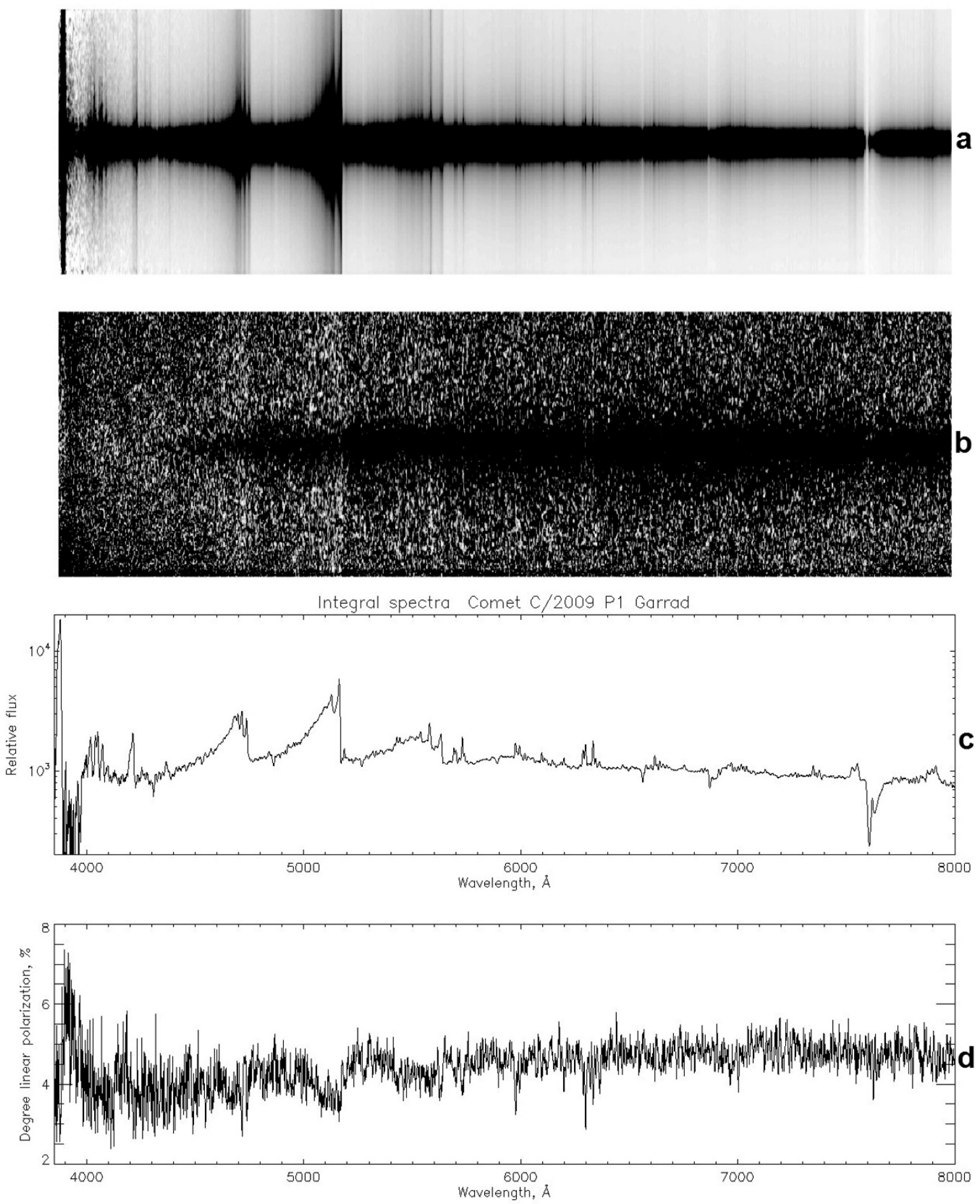

Fig. 2. Panels (a) and (b) display intensity and linear polarization for comet Garradd as a function of wavelength at a phase angle of $35.9^{\circ}$ on Feb 2 , 2012. Panels (c) and (d) display the integral intensity and the degree of linear polarization of the comet as a function of wavelength in the $3 \times 10$ arcsec area from the center of the comet. The position angle of the slit was $216.1^{\circ}$, implying that the slit was oriented perpendicularly to the extended Sun-comet radius vector.

\section{Results and Discussion}

\subsection{Linear polarization}

The phase-angle dependence of linear polarization obtained for comet Garradd is shown in Fig. 1. This figure also displays the value of linear polarization $P_{\mathrm{r}}=4.9$ $\pm 0.2 \%$ obtained from spectropolarimetric observations of the comet at the 6-m BTA telescope on February 2, 2012.
The polarization degree was averaged over a $3 \times 10$ arc$\sec (3336 \times 11118 \mathrm{~km})$ area centered at the nucleus in the $\mathrm{R}$ band of the spectrum. For comparison, the figure also shows the mean phase-angle dependence of polarization for dusty comets (i.e., comets with a high dust-to-gas ratio) (Mishchenko et al., 2010 and references therein), the polarization data and their fit for the unusual dusty comet C/1995 
C/2009 P1 Garrad, 2012-02-14, BTA+SCORPIO, filter 6840/40, total exposure $660 \mathrm{~s}$
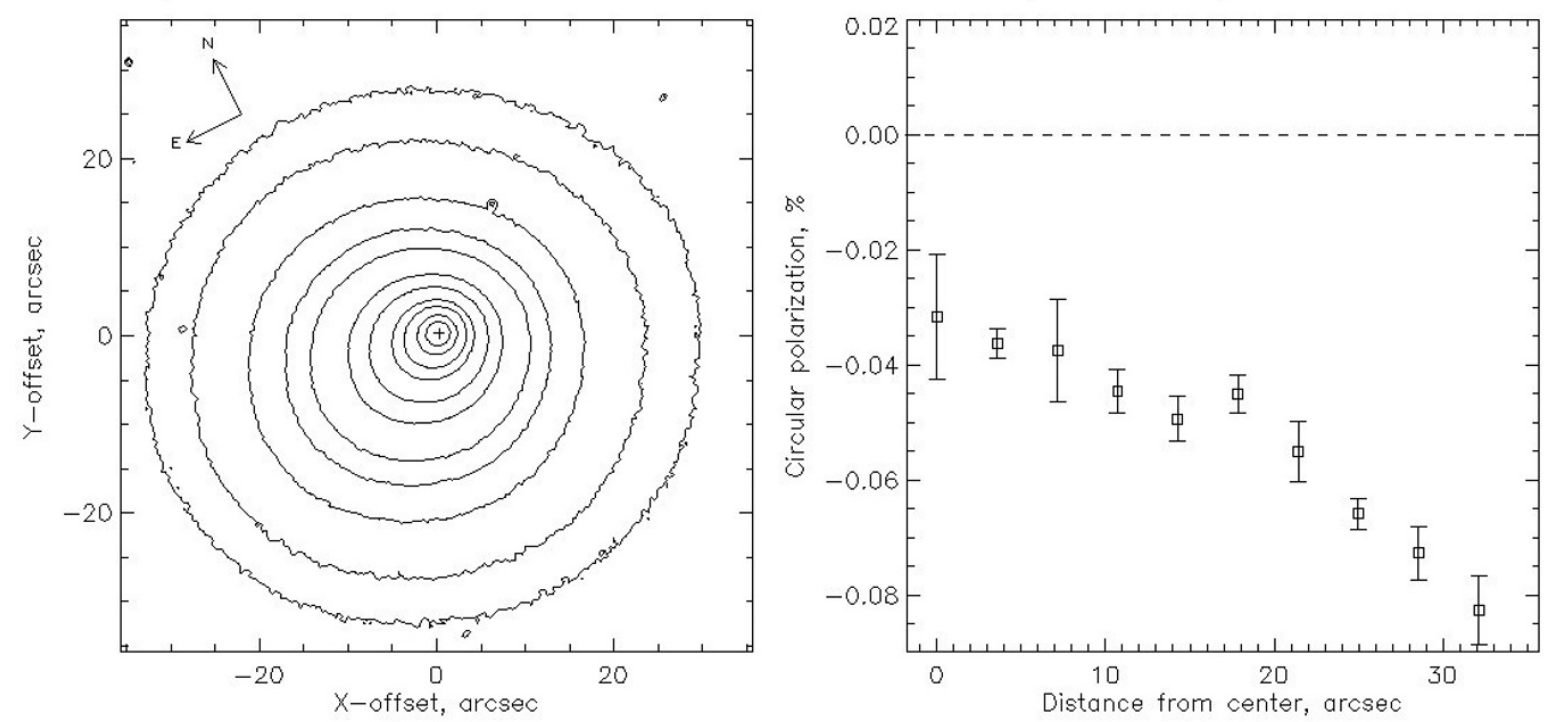

C/2009 P1 Garrad, 2012-04-21, BTA+SCORPIO, filter 6840/40, total exposure $720 \mathrm{~s}$
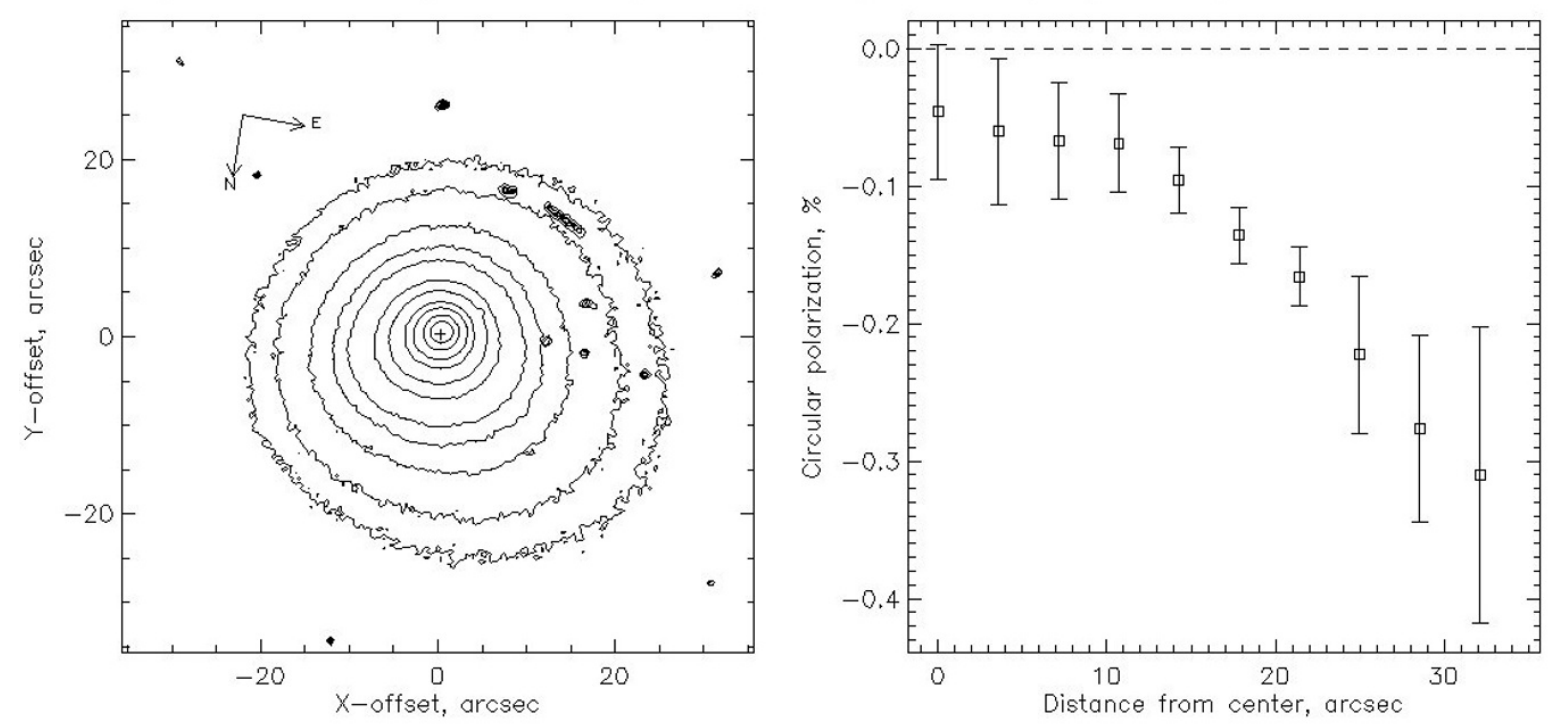

Fig. 3. Distribution of the intensity (left panel) and degree of circular polarization (right panel) over the coma of comet Garradd on February 14, 2012 (upper panel) and April 21, 2012 (bottom panel) in the red continuum filter 6840/90 $\AA$. The intensity contours are at $0.03,0.04,0.06,0.08,0.1,0.15$, $0.2,0.3,0.4,0.6,0.8$ of the maximum flux for the two observation dates. The robust average circular polarization in 20-px-wide ( $\sim 7^{\prime \prime}$ at the comet) circular apertures is shown as a function of the distance from the nucleus of the comet. The angle of 1 arcsec corresponds to $1005 \mathrm{~km}$ and $1424 \mathrm{~km}$ at the comet on February 14 and April 21, respectively.

O1 (Hale-Bopp) (Kiselev and Velichko, 1997), and phaseangle dependence of polarization for molecular emissions (Mishchenko et al., 2010 and references therein).

The scattered light from comets is the superposition of radiation from dust particles and gas molecules. Because of the complexity of cometary spectra, it is difficult to identify the continuum completely free of molecular emissions (Arpigny, 1995), especially for broad-band filters. At phase angles close to $90^{\circ}$, the polarization of light scattered by dust is $25^{\circ}$ (Kiselev and Rosenbush, 2004) and is much greater than that for resonance fluorescence of molecules, $\sim 8 \%$ (Le Borgne and Crovisier, 1987). Thus, a gaseous contamination causes depolarization of the observed continuum. The contamination is small for dusty comets, but can be significant for gassy comets (i.e., comets with a low dustto-gas ratio), such as comet Encke (Jockers et al., 2005). Moreover, the dust-to-gas ratio for comets is larger in the near-nucleus region of the coma. All of this leads to significant scatter of polarization data from $\sim 8 \%$ to $\sim 20 \%$ at large phase angles $\left(\alpha \sim 90^{\circ}\right)$ for comets with low dust-togas ratios (see, e.g., figure 4.2 in Mishchenko et al., 2010). Therefore, there is no good fit for polarization data of the gassy comets, while for the majority of dusty comets the phase dependence of polarization can be represented by the average curve.

Furthermore, some comets exhibit activity in the nearnuclear region of coma in the form of jets, the polarization of which can be higher (Hadamcik et al., 2013). The 
aperture measurements partly level the difference. In any case, because of differences in activity and dust/gas ratio, or other causes, the linear polarization value of comets may be highly dependent on the aperture.

As one can see in Fig. 1, the aperture polarization of comet Garradd (taking into account the observational errors) is close to that for dusty comets in the range of the observed phase angles $14^{\circ}-31^{\circ}$. The minimal degree of polarization is $P_{\mathrm{r}}=-2.2 \pm 0.2 \%$ at the phase angle $13.7^{\circ}$, while the average minimum of the negative polarization for dusty comets is $\sim-1.5 \%$ at a phase angle of $\sim 10^{\circ}$ (Kiselev and Rosenbush, 2004). Unfortunately, our data at $\sim 25^{\circ}-$ $31^{\circ}$ pertain to the phase angles where the polarization for dust differs slightly from that for gas emissions. Based on these aperture observations, nothing can be said about the depolarizing effect of the gas. Nevertheless, spectropolarimetric observations performed at the phase angle $35.9^{\circ}$ with the 6-m telescope on February 2 showed that the degree of continuum polarization is considerably larger than that for molecular bands. Therefore, on the basis of all polarization data, comet Garradd can be classified as being dusty. This is confirmed by direct spectroscopic data. The intensity and linear polarization degree for comet Garradd as a function of wavelength are shown in Figs. 2(a) and (b). Panels (c) and (d) display the integral intensity and the degree of linear polarization of the comet as a function of wavelength in the $3 \times 10$ arcsec area from the center of the comet. As one can see, the comet had a strong continuum and relatively weak $\mathrm{C}_{2}, \mathrm{C}_{3}$, and $\mathrm{NH}_{2}$ emissions. A small depolarization of continuum due to the $\mathrm{C}_{2}, \mathrm{C}_{3}$, and $\mathrm{NH}_{2}$ emission contamination can be seen in panel (d). The degree of polarization increases weakly with wavelength, which is typical of the majority of dusty comets at these phase angles (Kiselev et al., 2008).

\subsection{Circular polarization}

Figure 3 (left panels) shows the relative distribution of intensity over the coma of comet Garradd in the red continuum filter 6840/90 $\AA$ on February 14 (upper panel) and April 21 (bottom panel) of 2012. The intensity contours are at $0.03,0.04,0.06,0.08,0.1,0.15,0.2,0.3,0.4,0.6,0.8$ of the maximum flux for the two observation dates. The right-hand panels show robust average circular polarization in 20-px-wide ( $\sim 7^{\prime \prime}$ at the comet) circular apertures as a function of the distance from the nucleus of the comet. As one can see, the circular polarization for comet Garradd is left-handed (negative). By definition, circular polarization is left-handed $\left(-P_{\mathrm{c}}\right.$, negative) when the electric vector rotates anticlockwise, as viewed by an observer looking in the direction of light propagation (Mishchenko et al., 2002). The observed values of $P_{\mathrm{c}}$ vary noticeably over the coma (increasing in absolute value towards the periphery of the coma) in the ranges from $\sim-0.03$ to $\sim-0.08 \%$ and from $\sim-0.05$ to $\sim-0.3 \%$ on February 14 and April 21, respectively. The results obtained are in a good agreement with those for comets Halley, Hale-Bopp, S4 (LINEAR), Q4 (NEAT), Schwassmann-Wachmann 3, Tuttle, and Tempel 1 (Rosenbush et al., 2008 and references therein). The detection of left-handed circular polarization for the comet Garradd has confirmed our previous conclusion that the observed circular polarization for comets is predominantly left-handed (Rosenbush et al., 2007, 2008).

There are three mechanisms potentially responsible for circular cometary polarization: (a) multiple scattering in an asymmetric medium; (b) scattering by non-spherical aligned particles; and (c) scattering by optically-active particles. These mechanisms are discussed in detail by Rosenbush et al. (2007; see also references therein). The latter mechanism is of a special interest. In this case, circular polarization results from intrinsic properties of the dust particles, e.g., chirality of its molecules. However, all the above mechanisms face difficulties when applied to describe the circular polarization for comets. Although cometary circular polarization may shed light on fundamental processes of planetary and life formation, observations of cometary circular polarization are still extremely rare. This factor enhances the value of observations of each new comet, especially those performed with more advanced techniques.

Acknowledgments. The observations at the 6-m BTA telescope were obtained with the financial support of the Ministry of Education and Science of Russian Federation (state contracts no. 16.552.11.7028, 16.518.11.7073). The authors also express appreciation to the Large Telescope Program Committee of the RAS for the possibility of implementing the program of spectropolarimetric observations at the BTA. N. Kiselev is thankful to SOC and LOC of the conference "Cosmic Dust V" for financial support. We thank R. Schulz and D. Schleicher for the narrow-band cometary filters.

\section{References}

Afanasiev, V. L. and V. R. Amirkhanyan, Technique of polarimetric observations of faint objects at the 6-m BTA telescope, Astrophys. Bull., 67, 438-452, 2012.

Afanasiev, V. L. and A. V. Moiseev, The SCORPIO Universal Focal Reducer of the 6-m Telescope, Astron. Lett., 31, 194-204, 2005.

Afanasiev, V. L. and A. V. Moiseev, Scorpio on the $6 \mathrm{~m}$ telescope: current state and perspectives for spectroscopy of galactic and extragalactic objects, Baltic Astron., 20, 363-370, 2011.

Arpigny, C., Spectra of comets: Ultraviolet and optical regions, Laboratory and Astronomical High Resolution Spectra, ASP Conf. Ser., 81, 362382, 1995.

Chernova, G. P., N. N. Kiselev, and K. Jockers, Polarimetric characteristics of dust particles as observed in 13 comets-Comparisons with asteroids, Icarus, 103, 144-158, 1993.

Hadamcik, E., A. K. Sen, A. C. Levasseur-Regourd, R. Gupta, J. Lasue, and R. Botet, Dust in Comet 103P/Hartley 2 coma during EPOXI mission, Icarus, 222, 774-785, 2013.

Harrington, D. M., K. Meech, L. Kolokolova, J. R. Kuhn, and K. Whitman, Spectropolarimetry of the Deep Impact target Comet 9P/Tempel 1 with HiVIS, Icarus, 187, 177-184, 2006.

Hsu, J.-C. and M. Breger, On standard polarized stars, Astrophys. J., 262 732-738, 1982.

Jockers, K., N. Kiselev, T. Bonev, V. Rosenbush, N. Shakhovskoy, S. Kolesnikov, Yu. Efimov, D. Shakhovskoy, and K. Antonuyk, CCD imaging and aperture polarimetry of comet $2 \mathrm{P} /$ Encke. Are there two polarimetric classes of comets?, Astron. Astrophys., 441, 773-783, 2005.

Kiselev, N. N. and F. P. Velichko, Aperture polarimetry and photometry of Comet Hale-Bopp, Earth Moon Planets, 78, 347-352, 1997.

Kiselev, N. N. and F. P. Velichko, Polarimetry and Photometry of Comet C/1996 B2 Hyakutake, Icarus, 133, 286-292, 1998.

Kiselev, N. and V. Rosenbush, Polarimetry of comets: Progress and problems, in Photopolarimetry in Remote Sensing, edited by Videen, G. et al., 411-430, 2004.

Kiselev, N., V. Rosenbush, L. Kolokolova, and K. Antonyuk, The anomalous spectral dependence of polarization in comets, J. Quant. Spectrosc. Radiat. Transfer, 109, 1384-1391, 2008.

Kiselev, N., S. Velichko, K. Jockers, V. Rosenbush, and S. Kikuchi, Database of comet polarimetry, NASA Planetary Data System, EARC-COMPIL-5-DB-COMET-POLARIMETRY-V1.0, 2010.

Le Borgne, J. F. and J. Crovisier, Polarization of molecular fluorescence 
bands in comets: recent observations and interpretation, ESA Special Publ., 278, 171-175, 1987.

Mishchenko, M. I., L. D. Travis, and A. A. Lacis, Scattering, Absorption, and Emission of Light by Small Particles, 445 pp, Cambridge Univ. Press, New York, 2002.

Mishchenko, M. I., V. K. Rosenbush, N. N. Kiselev, D. F. Lupishko, V. G. Kaydash, I. N. Belskaya, Yu. S. Efimov, and N. M. Shakhovskoy, Polarimetric Remote Sensing of Solar System Objects, 291 pp, Akademperiodika, Kyiv, 2010.

Rosenbush, V., L. Kolokolova, A. Lazarian, N. Shakhovskoy, and N. Kiselev, Circular polarization in comets: observations of comet C/1999 S4 (LINEAR) and tentative interpretation, Icarus, 186, 317-330, 2007.

Rosenbush, V., N. Kiselev, and L. Kolokolova, Predominantly left-handed circular polarization in comets: Does it indicate L-enantiomeric excess in cometary organics?, in Organic Matter in Space, Proceedings of the International Astronomical Union, IAU Symposium, 251, 311-312, 2008.

Serkowski, K., Polarimeters for optical astronomy, in Planets, Stars and Nebulae, Studied with Photopolarimetry, edited by Gehrels, T., 135174, Univ. of Arizona Press, Tucson, 1974.

Shakhovskoy, N. N. and Yu. S. Efimov, Polarization observations of nonstable stars and extragalactic objects. I. Equipment, method of observation and reduction, Izv. Krymskoi Astrofiz. Obs., 45, 90-110, 1972.

N. N. Kiselev (e-mail: kiselevnn@yandex.ru), V. K. Rosenbush, V. L. Afanasiev, S. V. Kolesnikov, S. V. Zaitsev, and D. N. Shakhovskoy 